\title{
Fumarate Copolymer-Chitosan Cross-Linked Scaffold Directed to Osteochondrogenic Tissue Engineering
}

\author{
María Laura Lastra, María Silvina Molinuevo,, Ana María Cortizo, \\ María Susana Cortizo
}

Natural and synthetic cross-linked polymers allow the improvement of cytocompatibility and mechanical properties of the individual polymers. In osteochondral lesions of big size it will be required the use of scaffolds to repair the lesion. In this work a borax cross-linked scaffold based on fumarate-vinyl acetate copolymer and chitosan directed to osteochondrondral tissue engineering is developed. The cross-linked scaffolds and physical blends of the polymers are analyzed in based on their morphology, glass transition temperature, and mechanical properties. In addition, the stability, degradation behavior, and the swelling kinetics are studied. The results demonstrate that the borax cross-linked scaffold exhibits hydrogel behavior with appropriated mechanical properties for bone and cartilage tissue regeneration. Bone marrow progenitor cells and primary chondrocytes are used to demonstrate its osteo- and chondrogenic properties, respectively, assessing the osteoand chondroblastic growth and maturation, without evident signs of cytotoxicity as it is evaluated in an in vitro system.

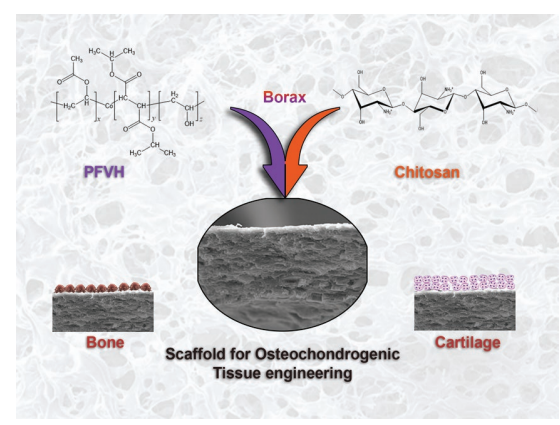

\section{Introduction}

Ostochondral tissue designs a special interfacial zone between bone and cartilage which is highly organized and plays a critical role in maintaining the cartilage tissue. The a-vascular nature of cartilage tissue is the reason for the

M. L. Lastra, Prof. M. S. Cortizo

Instituto de Investigaciones Fisicoquímicas

Teóricas y Aplicadas (INIFTA)

Facultad de Ciencias Exactas

Universidad Nacional de La Plata (UNLP)

CCT- La Plata, CONICET CC 16, Suc. 4., Argentina

M. L. Lastra, Dr. M. S. Molinuevo, Prof. A. M. Cortizo

Laboratorio de Investigaciones

en Osteopatías y Metabolismo Mineral (LIOMM)

Departamento de Ciencias Biológicas

Facultad de Cs. Exactas

Universidad Nacional de La Plata

47 y 115 (1900) La Plata, Argentina

E-mail: silvinamolinuevo@yahoo.com.ar poor regenerative capacity after injury or degenerative diseases. ${ }^{[1]}$ After damage osteochondral tissue often requires surgical procedures for the regeneration of the lesions. The surgical approach depends on the size of the lesion, but for lesions larger than $2.5 \mathrm{~cm}^{2}$ it is necessary the use of some biomaterial is needed to promote tissue regeneration. The materials proposed to be used in osteochondral tissue reparation include protein derived polymers (i.e., collagen, fibrin), carbohydrate-based matrices (i.e., alginate, agarose, chitosan) or synthetic polymers (usually polyesters, polyfumarates). ${ }^{[2,3]}$ Although most of the biomaterials used are based on homopolymers some authors proposed physical mixtures or cross-linked polymers. ${ }^{[2,4]}$ Natural polymers are a matter of intense research because of their high biocompatibility and low toxicity. However, their weak mechanical properties and the noncontrolled degradation rate have led to the design of composite materials between synthetic and natural polymers. ${ }^{[3,5,6]}$

Chitosan is a natural polymer derived from chitin, widely studied for biomedical applications. ${ }^{[7-11]}$ The 
choice of chitosan for tissue engineering is supported by its chemical nature, which in turn provides many possibilities for covalent and ionic modification that allows the adjustment of its mechanical and biological properties. $^{[10]}$ Different approaches to improve the physicochemical properties of chitosan were developed, i.e., blending it with natural or synthetic polymers. Blending of two or more polymers has gradually become an important approach to develop new biomaterials since no individual polymer completely meets the requirements of the tissue. As a consequence the resulting mix of polymers exhibits properties which are a combination of their primary material properties. ${ }^{[12]}$ However, in most cases there is some degree of incompatibility between the polymers that produce a blend of poor quality. In such cases, one strategy to improve the materials compatibility is polymer cross-linking achieved by the use of cross-linkers. ${ }^{[11,13-17]}$

In the present study we developed and characterized a new scaffold which was obtained by the cross-linking between a fumarate-based copolymer and chitosan. We studied its mechanical and swelling behavior as well as its aqueous stability and enzymatic-mediated degradation pattern. We also evaluated the scaffold in vitro biocompatibility using bone marrow progenitor cells (BMPC) and primary chondrocytes to evaluate cell growth kinetic on the scaffold as well as osteogenic and chondrogenic differentiation markers.

\section{Experimental Section}

\subsection{Materials}

Vinyl acetate (VA, 99\%), isopropyl alcohol, and benzoyl peroxide (BP, recrystallized from methanol) were purchased from Merck (Buenos Aires, Argentina). Fumaric acid was gifted by Maleic S.A., Argentina. Diisopropyl fumarate (DIPF) monomer was prepared and purified as previously described. ${ }^{[18]}$ Borax, Timper Laboratorios (99.9\%) and other solvents were supplied by from Merck and Sintorgan. Chitosan (Sigma-Aldrich, high molecular weight) was used as received. The degree of acetylation (DA) of chitosan, was assessed by Fourier transform infrared (FTIR) based on the absorbance ratio at 1320 and $1456 \mathrm{~cm}^{-1}$, corresponding to amide III and $\mathrm{CH}_{2}$ bands, respectively, as suggested by Brugnerotto et al. ${ }^{[19]}$

$$
\mathrm{DA}(\%)=31.92\left(\mathrm{~A}_{1320} / \mathrm{A}_{1456}\right)-12.20
$$

The viscosity average molecular weight $(M \eta)$ of chitosan was evaluated by capillary viscometry using acetic acid $0.3 \mathrm{M} /$ sodium acetate $0.2 \mathrm{~m}$ buffer as solvent. The measurement temperature was kept at $25.00 \pm 0.02{ }^{\circ} \mathrm{C}$. The $M \eta$ was evaluated in Equation (2)

$$
[\eta]\left(\frac{\mathrm{mL}}{\mathrm{g}}\right)=K M_{\eta}^{\alpha}
$$

where $[\eta]$ is the intrinsic viscosity and $K$ and $\alpha$ are characteristic parameters for each polymer-solvent system. $K$ and $\alpha$ parameters take values of $0.082 \mathrm{~mL} \mathrm{~g}^{-1}$ and 0.76 , respectively. ${ }^{[20]}$ Thus, chitosan exhibited $M \eta$ of $511 \mathrm{kDa}$ with $24 \%$ of DA.

\subsection{Fumarate Copolymer Synthesis}

Radical copolymerization of VA with DIPF, was carried out in bulk, initiated by BP under microwave energy, following the methodology previously reported. ${ }^{[21]}$ Briefly, both monomers (DIPF:VA, 75:25) together with the previously weighed mass of the initiator $\left(40 \times 10^{-3} \mathrm{M}\right)$ were charged into a reaction vessel and then purged with $\mathrm{N}_{2}$ during $30 \mathrm{~min}$. Reaction vessels were irradiated at $140 \mathrm{~W}$ during $25 \mathrm{~min}$ using a microwave oven (Zenith, ZVP-2819) of $2450 \mathrm{MHz}$ microwave frequency and $700 \mathrm{~W}$ maximum power. After reaching room temperature, the copolymer was isolated by hexane addition, purified by solubilization-precipitation (chloroform:hexane, 1:7), and then dried at constant weight for conversion estimation. The copolymer was designed as diisopropyl fumarate-vinyl acetate copolymer (PFV).

In order to introduce a hydroxyl group in to the main macromolecular chain, PFV was submitted to basic hydrolysis. To this end, the polymer was suspended in methanol (15\% wt/v) and $1 \% \mathrm{wt} / \mathrm{v} \mathrm{NaOH}$ solution was added ( $50 \mathrm{~mL} \mathrm{NaOH} / 15 \mathrm{~g}$ polymer); then the solution was stirred at $50{ }^{\circ} \mathrm{C}$ for $1 \mathrm{~h}$. After the reaction, the methanol was evaporated under vacuum, and the resulting solid was washed with water and dried until constant weight. This copolymer was designed as diisopropyl fumarate-vinyl acetate copolymer hydrolized (PFVH).

\subsection{Scaffolds Preparation}

To obtain the scaffold a 50\% w/w mixture of chitosan (CHI, SigmaAldrich, high molecular weight) and the previously synthesized copolymer were cross-linked in situ with borax by adaptation of the methodology of Sreedhar et al. ${ }^{[22]}$ Briefly, a PFVH copolymer solution in acetic acid ( $1 \% \mathrm{wt} / \mathrm{v})$ was added to the same volume of $1 \% \mathrm{wt} / \mathrm{v}$ of chitosan in dilute acetic acid ( $3 \% \mathrm{v} / \mathrm{v})$ with continue agitation. Then, borax (4\% wt/wt of the mixture of PFVH/ CHI) was added in order to carry out the cross-link. Finally, the blend was sonicated for $5 \mathrm{~min}$ and the obtained gels were casted by evaporating the solvent at room temperature on Teflon molds or culture plates. After drying, the membranes were neutralized with $\mathrm{NaOH}(5 \% \mathrm{wt} / \mathrm{v})$. This sample was designed as PFVH-CHI-B. For comparison purposes, a physical mixture between PFVH and CHI was prepared. The obtained gels were casted by evaporating the solvent (acetic acid) at room temperature and dried under vacuum up to constant weight. For the experiments, the scaffolds were sterilized by UV exposure for $30 \mathrm{~min}$.

\subsection{Characterization of Materials}

${ }^{1} \mathrm{H}$ NMR spectra of polymers were recorded with aVariam-200 $\mathrm{MHz}$ (Mercury 200) at $35{ }^{\circ} \mathrm{C}$ in acetonitrile- $\mathrm{d}_{3}$ or acetone- $\mathrm{d}_{6}$ for PFV or PFVH, respectively. Tetramethylsilane was used as an internal standard.

The FTIR spectra of the polymer films were recorded on a Nicolet 380 FTIR spectrometer between 4000 and $400 \mathrm{~cm}^{-1}$ with a resolution of $4 \mathrm{~cm}^{-1}$ and 32 accumulated scans. Briefly, a polymer solution in chloroform (5.0 wt\%) was prepared and poured onto the sodium chloride window. Then the solvent was 
allowed to evaporate at room temperature, with the resulting film dried under vacuum until the weight remained constant. The EZ-OMNIC software was used to analyze the spectra.

The molecular weight distribution and the average molecular weights were determined by size exclusion chromatography (SEC) using a LKB-2249 instrument at $25{ }^{\circ} \mathrm{C}$. A series of four $\mu$-Styragel columns, ranging in pore size $10^{5}, 10^{4}, 10^{3}, 100 \AA$, was used with chloroform as an eluent. The sample concentration was 4-5 $\mathrm{mg} \mathrm{mL}^{-1}$ and the flow rate was $0.5 \mathrm{~mL} \mathrm{~min}^{-1}$. The polymer was detected by the carbonylic absorption of the ester group $(5.75 \mu \mathrm{m})$, using an infrared detector (Miram 1A Infrared Analyzer) and the calibration was done with poly(methyl methacrylate) standard supplied by Polymer Laboratories and Polysciences.

The fractured sections of the scaffolds were obtained in liquid nitrogen using a razor blade, immediately afterward they were sputter with gold and their morphology was examined using scanning electron microscopy (SEM; Phillips 505, The Netherlands), with an accelerating voltage of $20 \mathrm{kV}$. The images were analyzed using Soft Imaging System ADDAII. The scaffolds surface was also examined by SEM.

Glass transition temperatures $\left(T_{\mathrm{g}}\right)$ were measured using a differential scanning calorimeter (Shimadzu-TA60). Samples $\left(\approx 5 \mathrm{mg}\right.$ ) were weighed and scanned at $10{ }^{\circ} \mathrm{C} \mathrm{min}-1$ from -30 to

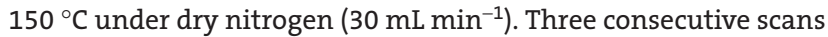
were performed for each sample: heating/cooling/heating.

\subsection{Stability and Degradation Assays}

The enzymatic degradation behavior of PFVH-CHI-B and PFVHCHI scaffolds was assessed by individually immersing the pre-weighed scaffolds in $10 \mathrm{~mL}$ phosphate-buffered saline (PBS, $0.01 \mathrm{M}, \mathrm{pH}$ 7.4) solution with or without $115 \mathrm{U} / \mathrm{L}$ lipase (EC 3.1.1.3, from Pseudomonas sp., Sigma) incubated at $37{ }^{\circ} \mathrm{C}$ for different periods of time $(7,15$, and $45 \mathrm{~d})$. All the prepared solutions were sterilized using a $0.2 \mu \mathrm{m}$ syringe filter and kept until usage at $4{ }^{\circ} \mathrm{C}$. For each condition a minimum of four samples was tested. All solutions were changed weekly. At the end of each degradation period, the samples were removed from the solution, rinsed with distilled water and weighted at constant weight.

\subsection{Mechanical Properties}

The tensile properties of the scaffolds were determined with a universal testing machine (DigimessTC500), using a force load cell ("Interface" of Arizona, USA, SM-50 N capacity) under controlled humidity conditions at $25{ }^{\circ} \mathrm{C}$ using a custom-build environmental chamber, following the methodology previously described. ${ }^{[23]}$ The dog bone shaped specimens $\left(50 \times 18 \mathrm{~mm}^{2}\right)$

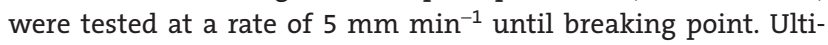
mate tensile stress, elastic modulus, and elongation at breaking point were calculated on the basis of the generated tensile stress-strain curves. The results presented are the mean values of eighteen independent measurements.

\subsection{Swelling Behavior}

The maximum swelling and water absorption capacity of the scaffolds were determined as it was previously reported. ${ }^{[24]}$ The water content of the membrane was obtained as the difference between $w$ and $w_{0}$, where $w$ was the weight of the water-saturated sample and $w_{0}$ was the weight of the initial dried sample. The percentage of swelling of the membrane was defined as

$$
\% S_{w}=\frac{100\left(w-w_{o}\right)}{w_{\circ}}
$$

In order to have insights into the water transport process through the scaffolds, the following equation was used to analyze the swelling process $^{[25]}$

$$
\frac{w_{t}}{w_{\infty}}=k t^{n}
$$

where $k$ is a characteristic constant of the system, which depends on the structural characteristics of the polymer and its interaction with the solvent, $n$ is the swelling exponent, which describes the mechanism of water transport into the membrane, while $w_{t}$ and $w \infty$ represent the quantities of water absorbed at time $t$ and at equilibrium time, respectively. In the above equation the numerical value of $n$ provides information about the water sorption mechanism. An $n$ value of 0.5 indicates Fickian kinetics where the rate of diffusion of water is the limiting process, while a value of $n$ between 0.5 and 1.0 indicates a non-Fickian diffusion process in which the relaxation of polymer chains determines the rate of water sorption. The limit case (designed as Case II transport where $n=1$ ) corresponds to a condition in which the rate of water diffusion is higher than the rate of polymer chain relaxation. In this condition the rate of mass uptake is directly proportional to time. ${ }^{[26]}$ The value of $n$ can be obtained from the slope, while $k$ is obtained from the interception of the plot of $\log \left(w_{t} / w \infty\right)$ versus log $t$ from the experimental data, taken until $60 \%$ of the maximum swelling.

\subsection{Biocompatibility Studies}

\subsubsection{Cell Cultures and Incubations}

For biocompatibility assays BMPC and chondrocyte cells were used. BMPC were isolated from the femora of Sprague-Dawley rats and cultured according to Molinuevo et al. ${ }^{[27]}$ Cells were maintained in basal media (DMEM-10\% FBS) at $37{ }^{\circ} \mathrm{C}$. Cell adhesion and proliferation was evaluated by the 3-(4,5-dimrthylthiazol2-yl)-2,5-diphenyl tetrazolium bromide (MTT) assay. This assay measures the reduction of the tetrazolium salt MTT to formazan by intact mitochondria in living cells. Thus, absorbance change is directly proportional to the number of viable cells. Briefly, $2.5 \times 10^{4}$ cells per well in basal media were plated onto the scaffolds which were casted on multiwell culture plates and cultured during different periods of time: $1 \mathrm{~h}$ (adhesion, proliferation $0 \mathrm{~d}$ ), or 1,3 , and $7 \mathrm{~d}$ (proliferation). After these culture periods, cells were incubated for two additional hours with a solution of $0.1 \mathrm{mg} \mathrm{mL}^{-1}$ MTT. After washing, the formazan precipitate was dissolved in dimethyl sulfoxide (DMSO) and the absorbance read at $570 \mathrm{~nm}$.

Alternatively, chondrocytes were isolated from the xiphoid process of the sternum of Sprague-Dawley adult rats after dissection of the perichondrium. Briefly, the cartilage was minced, washed three times in phosphate saline buffer $\mathrm{pH} 7.4$ (PBS), and treated with trypsin for $15 \mathrm{~min}$ at $37{ }^{\circ} \mathrm{C}$. After that cartilage was washed three times with DMEM-10\% FBS and maintained in culture at $37{ }^{\circ} \mathrm{C}$ under $95 \%$ air and $5 \% \mathrm{CO}_{2}$ with 
half of the culture media changed every $3 \mathrm{~d}^{[28]}$ After $28 \mathrm{~d}$, chondrocytes outgrowths from the cartilage were replated on the scaffolds and the mitogenic assay was conducted as described in the precedent paragraph.

All procedures were in accordance with the Guide for the Care and Use of Laboratory Animals published by the National Institutes of Health, and they were approved by the Institutional Laboratory Animal Care and Use Committee (CICUAL Protocol No. 001-05-15).

\subsubsection{Osteoblastic Differentiation}

Osteogenic induction of BMPC was performed by incubating the cells in basal media plus $\beta$-glycerol-phosphate and ascorbic acid during different periods of time as previously reported. ${ }^{[27]}$ After $15 \mathrm{~d}$ type I collagen was evaluated through the colorimetric assay with Sirius red and after $21 \mathrm{~d}$ mineral deposits were analyzed with the colorimetric assays of Alizarin S red.

\subsubsection{Chondroblastic Assays}

To evaluate the cartilage extracellular matrix produced by chondrocytes we determined the proteoglycans deposits after $21 \mathrm{~d}$ by the colorimetric method using alcian blue $\mathrm{pH}$ 3.5. Briefly, cells were fixed with formalin during $15 \mathrm{~min}$ and stained overnight with alcian blue $\mathrm{pH}$ 3.5. After washing, the stained material was detained with $4 \mathrm{~m}$ guanidine hydrochloride. The absorbance was determined at $570 \mathrm{~nm}$.

\subsubsection{Evaluation of Reverse Transcription-Polymerase Chain Reaction (RT-PCR) Gene Expression of Osteogenic and Chondrogenic Markers}

Total RNA was isolated from cultured stem cells and chondrocytes by the TRIZOL reagent method as suggested by the manufacturer (Invitrogen, Argentina). The RNA expression of osteogenic markers (alkaline phosphatase (ALP), Runx2, and type I collagen
(Col1a)) and chondrogenic markers (type II collagen (Col2), aggrecan, and SOX9) were analyzed using the semiquantitative RT-PCR using MMLV-RT (PB-L Productos Bio-Logicos, Argentina). All the markers were normalized using $\beta$-actin as housekeeping. The specific primers for the markers were designed from NCBI sequence data, using CLC Genomics Workbench software (OIAGEN) (Table 1) and band intensity was quantified using the gels plugin of MBF_ImageJ progam. The results of the PCR semiquantitative analysis were expressed as relative intensity, and represent the quotient of the signal intensity of a specific band of each marker and the signal intensity of $\beta$-actin as housekeeping.

\subsubsection{Evaluation of Cytotoxicity}

The eventual cytotoxicity of the biomaterial was evaluated by measuring nitric oxide (NO), interleukin-1 $\beta$ (IL1 $\beta$ ), and tumor necrosis factor $\alpha$ (TNF $\alpha$ ) production using a model of macrophages in culture. Briefly, RAW264.7 macrophages were grown on the scaffolds or the tissue culture plates (control condition) in DMEM without phenol red-5\% FBS. After 24, 48, and $72 \mathrm{~h}$ the supernatants were collected and evaluated for NO production by the Griess' assay. IL1 $\beta$ and TNF $\alpha$ production were evaluated by ELISA kits (BD OptEIA mouse IL-1 $\beta$ ELISA and BD OptEIA mouse TNF (Mono/Mono) ELISA set) in the conditioned media 2, 7, and $13 \mathrm{~d}$ of incubation.

\subsection{Statistical Analysis}

Results are expressed as the mean \pm SEM and, unless indicated otherwise, were obtained from two separate experiments performed in triplicate. Differences between groups were assessed by one-way ANOVA with Tukey post hoc test. For non-normal distributed data nonparametrical Kruskal-Wallis with Dunn's post hoc test was performed, using Graph Pad InStat v. 3.00 (Graph Pad Software, San Diego, CA, USA). $p<0.05$ was considered significant for all statistical analyses.

Table 1. Primer sequence for osteo- and chondrogenic markers.

\begin{tabular}{|c|c|c|c|c|}
\hline Marker & Genbank code & Product size (bp) & & Sequence \\
\hline \multirow[t]{2}{*}{$\beta$-actin } & NM_031144.3 & 345 & fw & ССТTCAACACCCCAGCCAT \\
\hline & & & rv & CATAGCTCTTCTCCAGGGA \\
\hline \multirow[t]{2}{*}{ RUNX2 } & XM_006244554.2 & 598 & fw & GCCGGGAATGATGAGAACTA \\
\hline & & & rv & TGAGAGAGGAAGGCCAGA \\
\hline \multirow[t]{2}{*}{ ALP } & J03572.1 & 737 & fw & GACAGCAAGCCCAAGAGA \\
\hline & & & rv & CAGTTCAGTGCGGTTCCA \\
\hline \multirow[t]{2}{*}{ Colla1 } & NM_053304.1 & 651 & fw & GCATACACAATGGCCTAA \\
\hline & & & rv & CTGTTCCAGGCAATCCAC \\
\hline \multirow[t]{2}{*}{ Col2 } & NM_012929.1 & 295 & fw & GGGCAGAAAGGAGAACCT \\
\hline & & & rv & TTGCATGACTCCCATCTGG \\
\hline \multirow[t]{2}{*}{ SOX9 } & NM_080403.1 & 503 & fw & TCTCCTGGACCCCTTCAT \\
\hline & & & rv & GGTGGTCTTTCTTGTGCT \\
\hline \multirow[t]{2}{*}{ Aggrecan } & NM_022190.1 & 525 & fw & CCATCCCCTGCTACTTCATC \\
\hline & & & rv & CACCATAGCAACCTTCCC \\
\hline
\end{tabular}




\section{Results and Discussion}

\subsection{Copolymer Synthesis and Characterization}

Based on our previous experience, we performed the synthesis of diisopropyl fumarate-vinyl acetate copolymer (PFV) by radical copolymerization under microwave conditions. The known advantage of such methodology was previously applied for other similar systems in our group, where we demonstrated that the rate of polymerization has been significantly faster than under thermal conditions. ${ }^{[18,21]}$ In the present case, the reaction conversion attaining at $140 \mathrm{~W}$ and $25 \mathrm{~min}$ of reaction time was $40 \%$. On the other hand, the selection of the comonomers was based on the prior knowledge of the properties of fumaric copolymers, such as hydrophobicity/hydrophilicity ratio, stability, and glass transition temperature. ${ }^{[29,30]}$ The structure of the obtained copolymer PFV starting of 75:25 (DIPF:VA) feed monomer compositions under microwave conditions were confirmed by FTIR and ${ }^{1} \mathrm{H}$ NMR. FTIR (thin film, $\mathrm{cm}^{-1}$ ): 2979, 2931, 2877 (C-H alifatic), 1730 (C=O), 1233 and 1106 (CO-OR). Figure 1 shows the ${ }^{1}$ H NMR spectrum including the structure and the peaks assignation, based on the corresponding homopolymers spectra. ${ }^{1} \mathrm{H}$ $\operatorname{NMR}\left(\mathrm{CD}_{3} \mathrm{CN}\right): \delta(\mathrm{ppm}), 1.26\left(-\mathrm{CH}_{3}\right), 1.97\left(-\mathrm{CH}_{2}-\right), 2.19$ $\left(\mathrm{CH}_{3}-\mathrm{CO}\right), 2.97(-\mathrm{CH}<), 4.98\left(-\mathrm{OCH}\left(\mathrm{CH}_{3}\right)_{2}\right)$.

Composition of PFV was estimated from the integral ratio of the peak at $1.26 \mathrm{ppm}$ corresponding to methyl hydrogen $\left(\mathrm{CH}_{3}\right)$ of DIPF unit and the peaks between 1.4 and $2.2 \mathrm{ppm}$ corresponding to methylene and methyl hydrogen $\left(\mathrm{CH}_{2}\right.$ and $\left.\mathrm{CH}_{3}\right)$ of VA unit, using the following ratio

$$
F_{\mathrm{DIPF}}=\frac{I_{1} / 12}{I_{1} / 12+I_{2} / 5}
$$

where $F_{\mathrm{DIPF}}$ is the mole fraction of DIPF in the copolymer and $I_{1}$ and $I_{2}$ represent the ${ }^{1} \mathrm{H}$ NMR resonance peak areas at $1.26 \mathrm{ppm}$ and near to $2.0 \mathrm{ppm}$, respectively. Based on this analysis, the $F_{\mathrm{DIPF}}$ of PFV was 0.68 . The weight-average molecular weight $\left(M_{\mathrm{w}}\right)$ and polydispersity index were $42600 \mathrm{~g} \mathrm{~mol}^{-1}$ and 6.4, respectively, evaluated through SEC according to the ratio $M_{\mathrm{w}} / M_{\mathrm{n}}$.

Then, PFV copolymer was partially hydrolyzed in order to increase its hydrophilicity for a better interaction with chitosan. It was formerly reported that acid and alkaline hydrolyses of poly(DIPF) to obtain poly(hydroxycarbonylmethylene) were very difficult, due to the steric effect of the bulky group on the macro- molecular chain. ${ }^{[31]}$ Thus, under the selected experimental conditions, only the VA ester groups can be hydrolyzed (see Scheme 1).

The structure of PFVH was demonstrated by FTIR and ${ }^{1} \mathrm{H}$ NMR spectroscopic analysis. FTIR (thin film, $\mathrm{cm}^{-1}$ ): $3438(\mathrm{O}-\mathrm{H}), 2979,2931,2877$ ( $\mathrm{C}-\mathrm{H}$ alifatic), 1730 (C=O), 1233 and 1106 (CO-OR), $666\left(\delta_{\text {out plane, }} \mathrm{O}-\mathrm{H}\right)$. Figure 2 shows the ${ }^{1} \mathrm{H}$ NMR spectrum including the structure and the peaks assignation. ${ }^{1} \mathrm{H} \mathrm{NMR}\left(\mathrm{CD}_{3} \mathrm{COCD}_{3}\right): \delta(\mathrm{ppm}), 1.32$ $\left(-\mathrm{CH}_{3}\right), 2.07\left(-\mathrm{CH}_{2}-; \mathrm{CH}_{3}-\mathrm{CO}\right), 2.96(\mathrm{HO}-\mathrm{CH}<;-\mathrm{CH}<)$, $5.03\left(-\mathrm{OCH}\left(\mathrm{CH}_{3}\right)_{2}\right)$. As it can be seen in this figure, there is a broad peak close to $3 \mathrm{ppm}$ similar to the peak that appears in the ${ }^{1} \mathrm{H}$ NMR spectrum of PFV. However, it is overlapped with another sharp peak assigned to the VA unit hydrolyzed together with the disappearance of $-\mathrm{CH}_{3}$ signal at $2.2 \mathrm{ppm}$. Both observations together with the $\mathrm{O}-\mathrm{H}$ band $\left(3438 \mathrm{~cm}^{-1}\right)$ of FTIR spectrum, confirm the efficiency of the hydrolysis reaction.

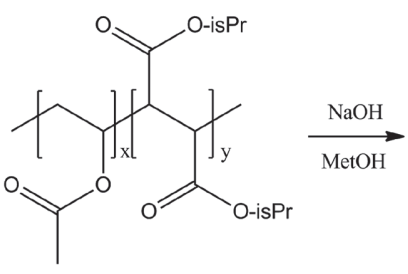

PFV

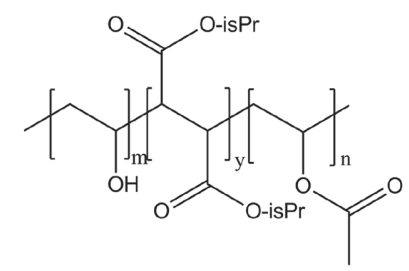

PFVH
Scheme 1. Hydrolysis reaction of PFV to produce PFVH. As it was described in the Experimental Section, hydrolysis reaction was carried out under basic methanolic conditions at $50^{\circ} \mathrm{C}$ during $1 \mathrm{~h}$. 


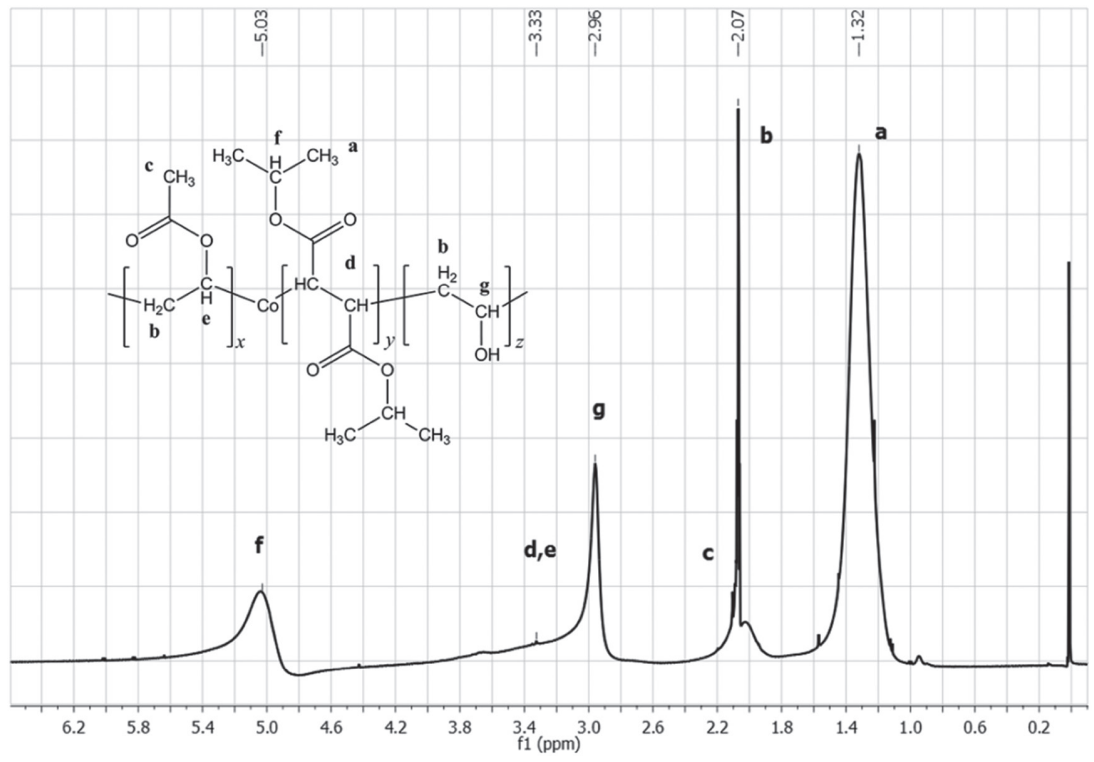

Figure 2. ${ }^{1} \mathrm{H}$ NMR spectrum of PFVH in acetone- $\mathrm{d}_{6}$ at $35^{\circ} \mathrm{C}$. The inset shows the corresponding copolymer structure with the peak assignments.

\subsection{Scaffolds Preparation and Characterization}

One of the most important issues related to the polymer blend preparation is its compatibility, which is closely related to the polymer-polymer interactions. In particular, numerous chitosan-based blends were prepared using different methodologies, among which we can highlight the use of cross-linking agents. For example, chitosan/ poly(vinyl alcohol) was cross-linked with borate, tripolyphosphate and glutaraldehyde. ${ }^{[13]}$ In the present work we have selected borate as cross-linker agent. This reagent is able to form a complex between tetrahydroxyborate ion and various polyols, a kind of interaction that it is not limited to hydroxyl groups on adjacent carbon atoms but can also involve those on alternate carbon atoms. ${ }^{[32]}$ Thus, we expect that borax act as cross-linker agent forming a complex between the hydroxyl groups of both polymers, $\mathrm{PFVH}$ and $\mathrm{CHI}$.

To evaluate polymer compatibility, we first studied the surface of the scaffold by SEM comparing the mixture of $\mathrm{PFVH}$ with chitosan (PFVH-CHI) before (Figure $3 \mathrm{~A}, \mathrm{~B}$ ) and after the addition of Borax (Figure $3 C, D$ ). We found that PFVHCHI scaffolds without borax have a scaly surface (Figure $3 \mathrm{~A}$ ) with evident signs of phase separation in the fracture edge (Figure 3B, arrow). On the other hand, the PFVH-CHI-B scaffold presented a rough homogeneous surface (Figure $3 \mathrm{C}$ ) and the same structure seems to continue in the breaking surface (Figure 3D).

The glass transition temperature $\left(T_{\mathrm{g}}\right)$ was determined by differential scanning calorimetry. Figure 4 show the diferential scanning calorimetry (DSC) analysis of PFV, PFVH copolymers, and PFVH-CHI-B sample including $4 \%$ of borax. PFVH exhibited an increase of $T_{\mathrm{g}}$ $\left(60.5^{\circ} \mathrm{C}\right)$ in comparison to PFV $\left(48.3^{\circ} \mathrm{C}\right)$, which can be attributed to a decrease in chain structure flexibility, due to intra/ interchain hydrogen bond. No modification of $T_{\mathrm{g}}$ was observed for the physical blend (PFVH-CHI, data no shown) compared to PFVH. On the other hand, the caloric behavior of PFVH-CHI-B sample is less clear but an increase in the $T_{\mathrm{g}}$ of $4.3{ }^{\circ} \mathrm{C}$ was observed. These results suggest that the borax produce the crosslinking between the PFVH and CHI polymers, which restricts the catenaries movements, as it was previously observed for other related systems. ${ }^{[13,33]}$

\subsection{Stability and Degradation}

We have also evaluated the stability and the possible degradation of the scaffolds in phosphate saline buffer
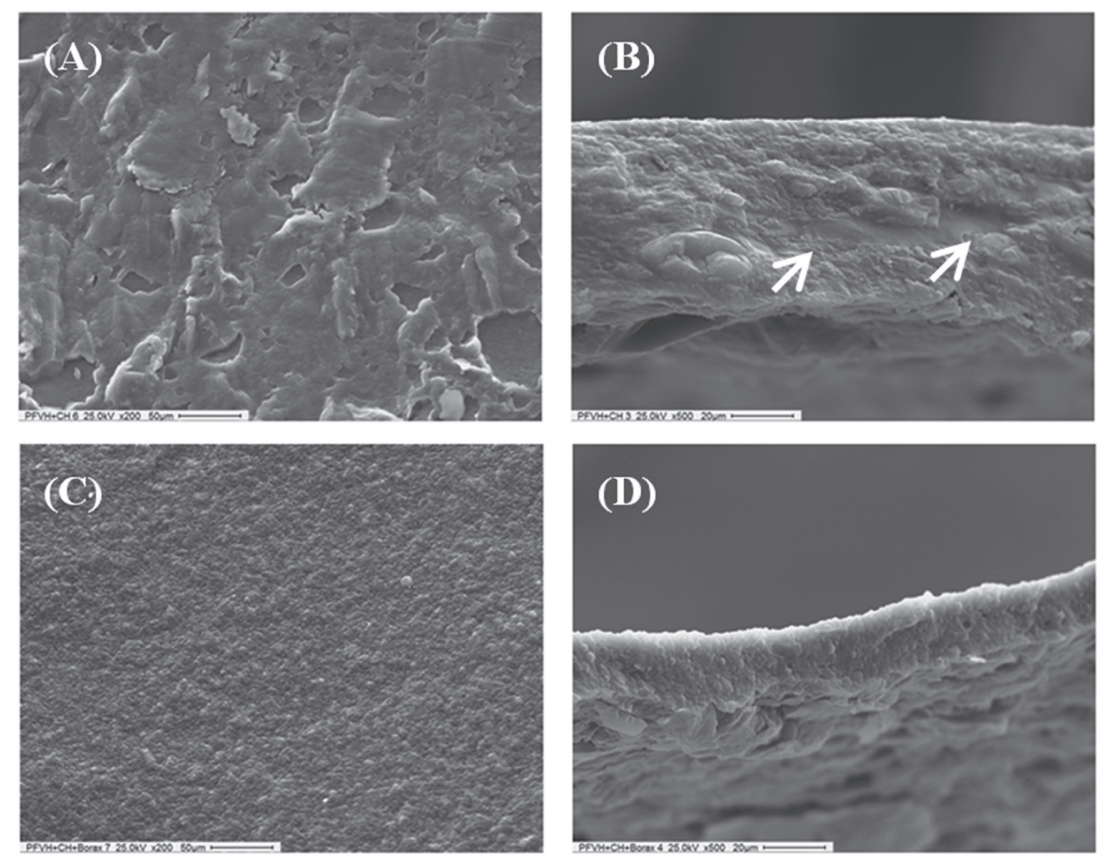

Figure 3. SEM images of PFVH-CHI and PFVH-CHI-B. The surface characteristics and the breaking surface of $\mathrm{A}, \mathrm{B}) \mathrm{PFVH}-\mathrm{CHI}$ or $\mathrm{C}, \mathrm{D}) \mathrm{PFVH}-\mathrm{CHI}-\mathrm{B}$ scaffolds. 


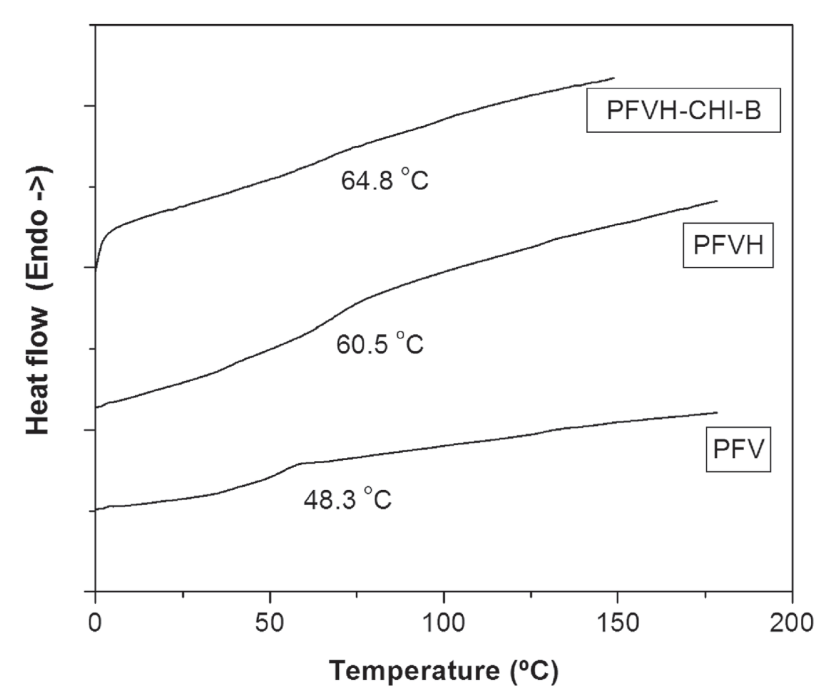

Figure 4. DSC thermograms of the copolymer PFV; PFVH, the hydrolysis product of PFV and the PFVH borax-cross-linked chitosan scaffold, PFVH-CHI-B.

$\mathrm{pH} 7.4$ at $37^{\circ} \mathrm{C}$. We found a linear increase in the weight loss for both PFVH-CHI and PFVH-CHI-B with a maximum of about $10 \%$ of weight loss after $45 \mathrm{~d}$ of incubation, without differences between PFVH-CHI and PFVH-CHI-B scaffolds (Figure 5A). However, the enzymatic degradation rate for PFVH-CHI-B scaffolds was significantly lower than the $\mathrm{PFVH}-\mathrm{CHI}$ rate during the short times $(7$ and $15 \mathrm{~d})$ but reaching similar levels at $45 \mathrm{~d}$ (Figure 5B). Moreover, the maximum \% of weight loss was similar to those obtained in PBS (Figure 5A). It is known that chitosan is susceptible to be degraded in vivo by lipase, which appear to target acetylated residues. ${ }^{[10,34]}$ This process is dependent on the acetylation degree of chitosan, where low degree of acetylated samples exhibited a relatively low degradation rate. In our system we can attributed the low degradation rate to chitosan, the only polymer which can suffer an enzymatic degradative process. This hypothesis could also explain the similar degradation behavior of the scaffolds under hydrolytic conditions, but also it allows us to hypothesize that the cross-linking borax is affecting the degradation rate of chitosan. It has been previously demonstrated in related blends of chitosan that their degradation can be attributed to the degradation of chitosan rather than to other compounds presents on the blends. ${ }^{[35]}$

\subsection{Mechanical Properties}

The mechanical performance of PFVH-CHI-B and PFVHCHI scaffold were analyzed by traction test. The tensile strength, elongation at break, and elastic modulus were $10 \pm 2 \mathrm{MPa}, 6.0 \% \pm 1.0 \%$, and $344 \pm 38 \mathrm{MPa}$, respectively, for PFVH-CHI-B. In comparison, the tensile strength, elongation at break, and elastic modulus for the PFVH-CHI
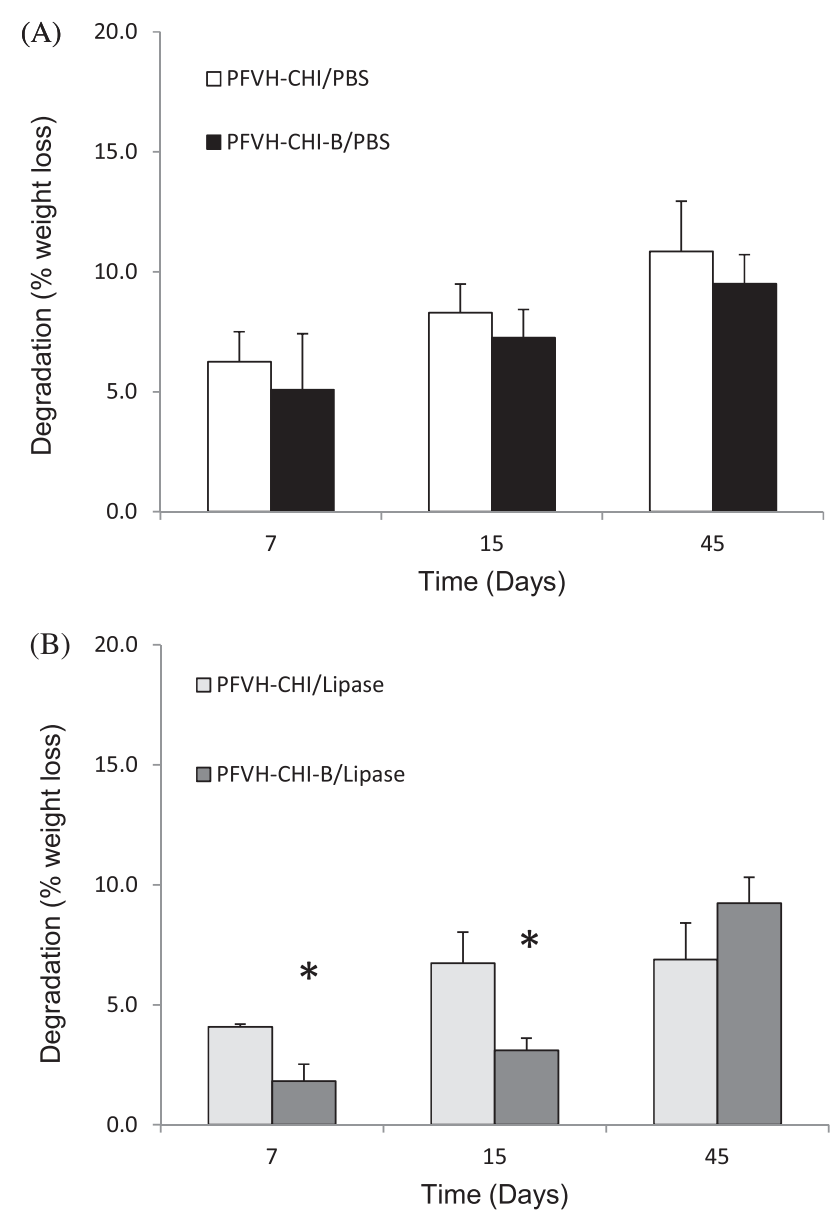

Figure 5. Scaffold degradation study. $\mathrm{PFVH}-\mathrm{CHI}$ and $\mathrm{PFVH}-\mathrm{CHI}-\mathrm{B}$ degradation behavior was evaluated in A) buffer $\mathrm{pH} \mathrm{7,4}$ and B) lipase solution at $37{ }^{\circ} \mathrm{C}$ during different periods of time. ${ }^{*} p<0.05$ versus $\mathrm{PFVH}-\mathrm{CHI}, n=5$.

scaffold were $11 \pm 2 \mathrm{MPa}, 5.6 \% \pm 0.7 \%$ and $407 \pm 30 \mathrm{MPa}$, respectively. There were no significant differences on these parameters studied between the scaffolds with or without borax, which could be explained by the low cross-linked rate of the networks because of the low borax proportion used in our matrices. These results are consistent with data previously showed in the DSC studies where small differences were found between the PFVH-CHI and PFVHCHI-B scaffolds. Moreover, our results are comparable to the mechanical properties of other structural related systems ${ }^{[13]}$ but compared to the articular cartilage they present the highest modulus due to the absence of intern porous, as it was proved by other researches. ${ }^{[36,37]}$

\subsection{Swelling}

Figure 6 presents the swelling kinetics of the PFVH-CHI-B scaffolds in buffered aqueous solutions of $\mathrm{pH} 7.4$ at $37^{\circ} \mathrm{C}$. In this figure it can be observed that the degree of swelling increases with time, until a certain point where it becomes 


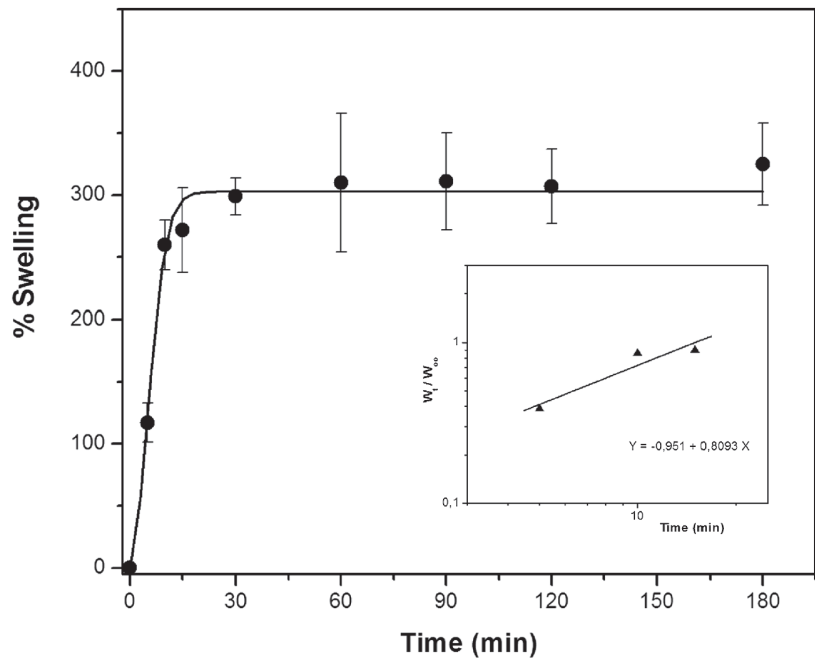

Figure 6. Swelling kinetic of the PFVH-CHI-B scaffold was studied in phosphate-saline buffer $\mathrm{pH} 7.4$ at $37^{\circ} \mathrm{C}$.

constant, reaching equilibrium within $30 \mathrm{~min}$. The maximum swelling attained was $303 \%$, which is a typical value for hydrogels. ${ }^{[38,39]}$

During the swelling process the water molecule diffuse into the polymer matrix acting as plasticizers and promoting the relaxation of the macromolecular chains. As it was previously mentioned, the kinetics of such process could be analyzed through the Fick model (Equation (4)) in order to determine the $n$ exponent. The inset in Figure 6 shows the linear regression plots of the fractional-water absorbed at short times according to Equation (4), from which the $n$ value of 0.809 could be calculated. This result suggests that the mechanism of water transport within the scaffold occurred by a non-Fickian diffusion process, probably with a rapid water diffusion in comparison to the macromolecular chain relaxation.

\subsection{Biocompatibility Studies}

Then, we evaluated the growth kinetic of BMPC and primary chondrocytes to investigate the biocompatibility of the PFVH-CHI-B scaffold (Figure 7A,B, respectively). We found that both cell lines growth in a similar way on the scaffold and on the tissue culture plates. However, the growth of BMPC was time-dependent with a saturation of the hyperbolic curve after $2 \mathrm{~d}$ of culture when cells are growing on culture dishes, while the cells growing on the scaffold continues growing up to $7 \mathrm{~d}$ (Figure 7A). On the other hand, the chondrocytes had a lag time and after that there was an exponential increase in cell number (Figure 7B).

Then we evaluated the capacity of BMPC to differentiate toward an osteoblastic phenotype when growing on the scaffold. We found that BMPC growing on the scaffolds produced significantly higher amounts of type I
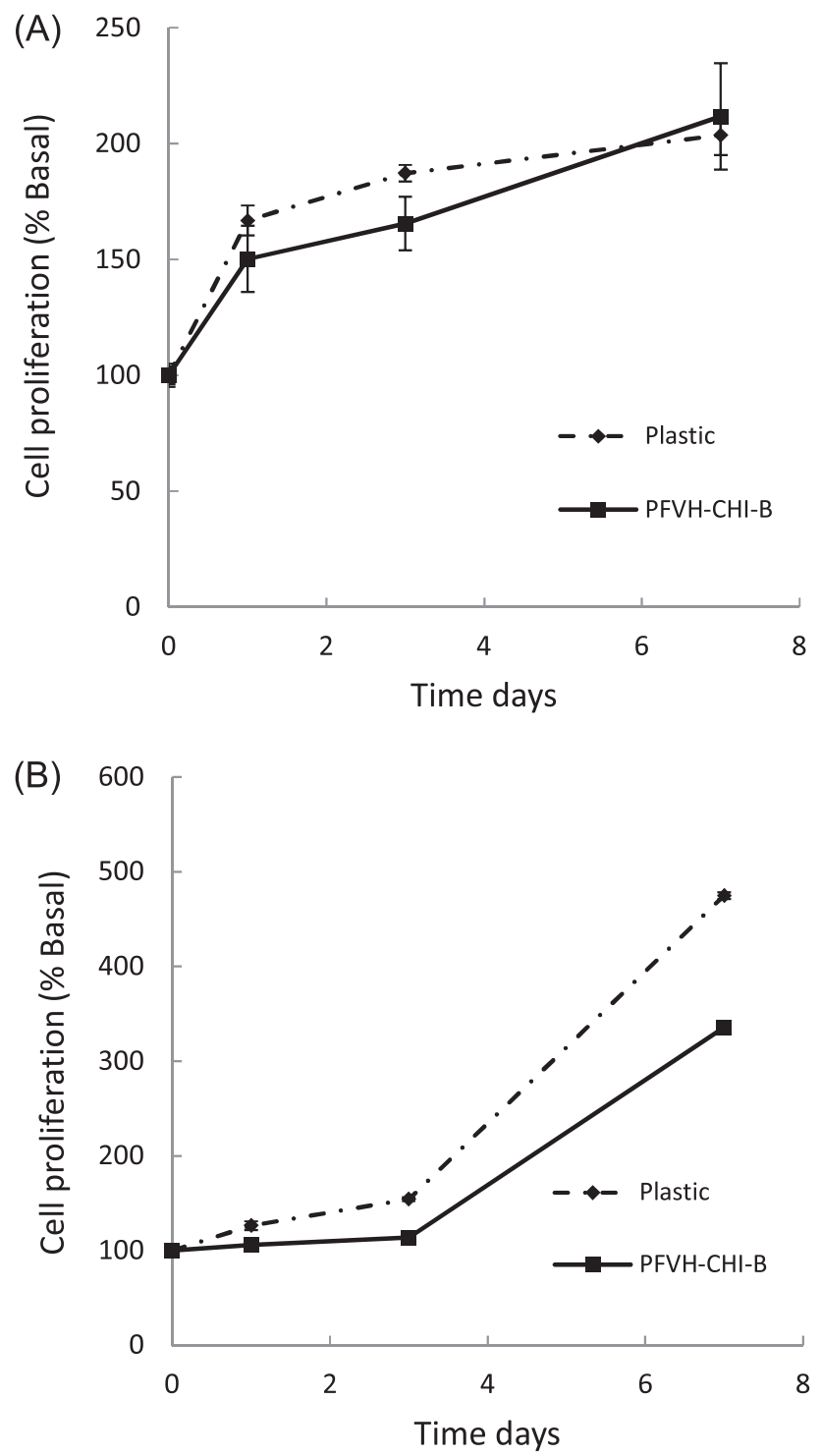

Figure 7. Cell proliferation assays. Time course of A) BMPC and B) chondrocyte cell growth was evaluated by MTT assay as it was described in the Experimental Section. Results are expressed as \% Basal (o d of proliferation), $n=6$.

collagen or mineral nodules after 15 or $21 \mathrm{~d}$, respectively, of osteoblastic differentiation compared to BMPC differentiated on the tissue culture plates (Figure 8A, $p<0.01$ ). We also evaluated the mRNA expression of different osteoblastic markers Runx2, ALP, and Col1a by RT-PCR. The analysis of the PCR bands showed that that the expression of all markers in the BMPC remain unchanged after $15 \mathrm{~d}$ of culture with osteogenic media on tissue culture dishes (plastic, P) or on the scaffold (SC) (Figure 8B,C).

Chondrocytes growing on the scaffold showed a denser disposition than those growing on tissue culture plates (Figure 9A). Additionally, we found that primary chondrocytes growing on the scaffold produced higher amounts of the proteoglycan matrix than cells growing 

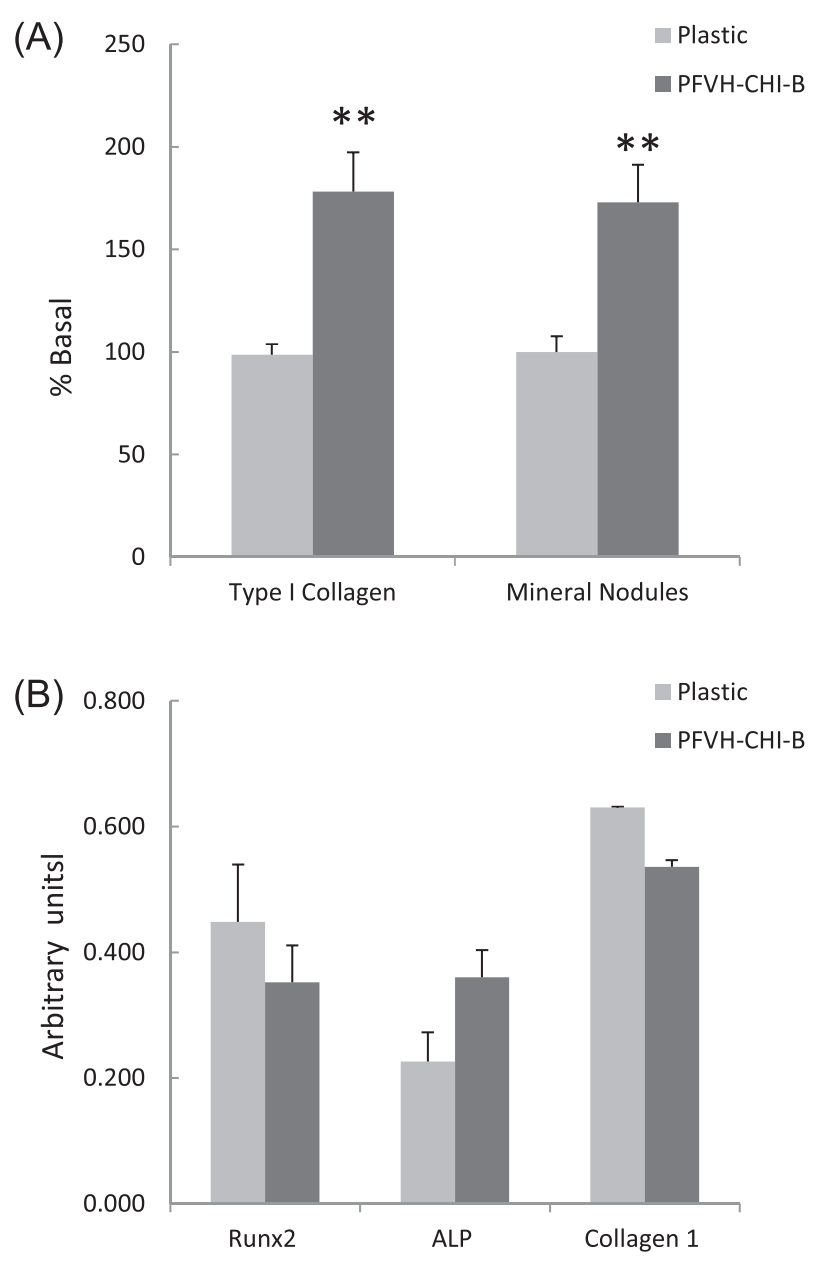

(C)

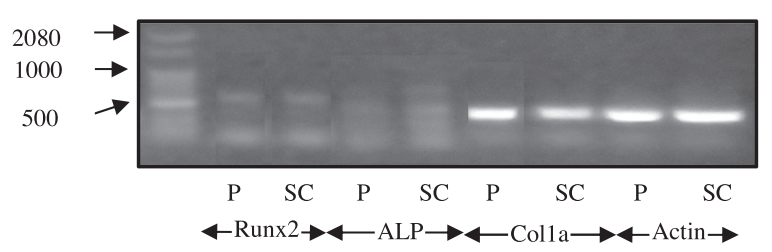

Figure 8. Osteoblastic differentiation. A) BMPC were cultured in an osteoblastic differentiation media and collagen type I (after $15 \mathrm{~d}$ ) or mineral nodules ( $21 \mathrm{~d}$ ) production were evaluated. B) Semiquantitative analysis of mRNA for Runx2, ALP, and collagen type I after $7 \mathrm{~d}$ of differentiation. C) The PCR products of Runx2, alkaline phosphatase, collagen type I, and actin for BMPC growing on plastic (P) or PVFH-CHI-B (SC), respectively. ${ }^{* *} p<0.01, n=5$.

on tissue culture plates (Figure 9B). Evaluation of the mRNA for chondroblastic markers showed that Col2 and SOX9 levels were similar for chondrocytes growing on both plastic and PVFH-CHI-B (SC). Contrary, cells growing on the PVFH-CHI-B scaffolds expressed significantly higher levels of aggrecan than the cells growing on plastic (Figure 9C,D, respectively, $p<0.05$ ). Thus, the scaffold-
(A)
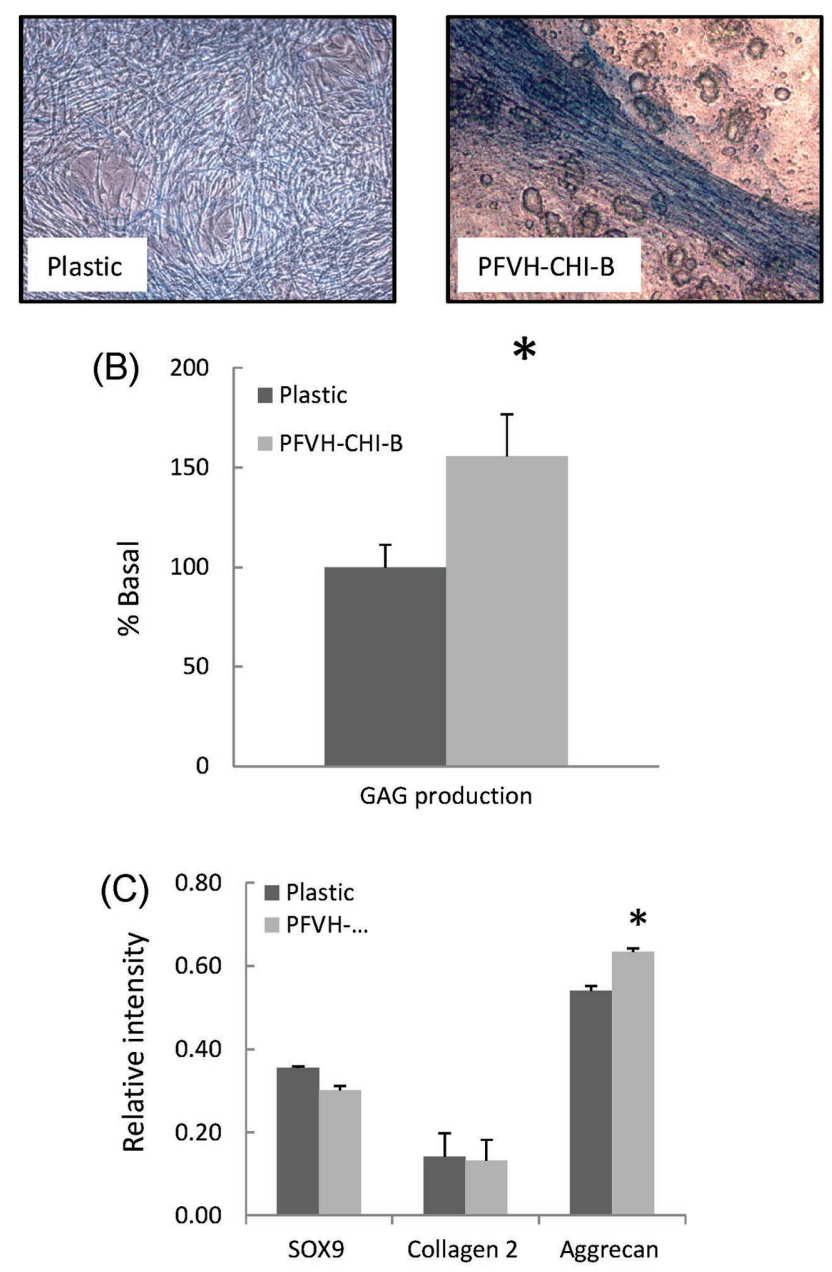

(D)

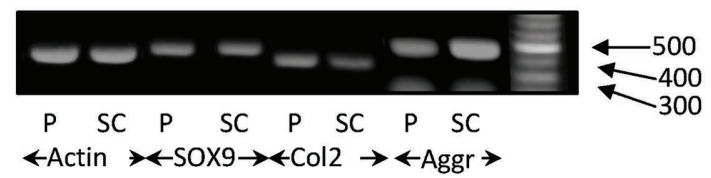

Figure 9. Chondrogenesis was evaluated by the assessment of glycosminoglycan (GAG) production. Primary chondrocytes growing on A) plastic or PVFH-CHI-B matrix were stained with alcian blue after $21 \mathrm{~d}$ of culture, and $\mathrm{B}$ ) the production of GAG was evaluated spectrophotometrically. The expression of molecular markers for chondrogenic development was evaluated by PCR after $10 \mathrm{~d}$ of culture $C, D)$ for chondrocytes growing on plastic $(P)$ or PVFH-CHI-B (SC). ${ }^{*} p<0.05, n=5$.

induced up-regulation of different markers could have different kinetics at mRNA levels.

Nevertheless, our results suggest that this novel biomaterial presents enough versatility to be used on the osteochondral interface regeneration. Previous data demonstrated that chitosan-based scaffolds presented good biocompatibility allowing both chondrocyte and osteoblast development. ${ }^{[40-43]}$ It has been previously 
reported that chitosan blends with synthetic polymers or ceramics improve osteo- or chondroblast growth and development. However, there are few reports about scaffolds that supports both osteoblastic and chondroblastic development. ${ }^{[11,43]}$ In this sense, our scaffold represents an improving on the chitosan-based biomaterials because it allows the in vitro development and differentiation of both osteoblasts and chondrocytes.

\subsection{Cytotoxicity Studies}

Finally, we also investigate if our scaffolds might generate any cytotoxic effect using RAW264.7 macrophages in culture and evaluating the cytokine (NO, IL1 $\beta$, and TNF $\alpha$ ) production during different times. Macrophages have the capacity to react toward foreign bodies producing inflammation mediators such as IL, TNF $\alpha$, and NO, and thus represent a good model to investigate the cytotoxicity of biomaterials. In the present work, we found no differences on nitric oxide production after the cells were grown on the scaffolds or in control condition (standard culture dishes) (Table 2). Similar results were obtained for IL1 $\beta$ and TNF $\alpha$ (Table 3). Although there was an increase in cytokine secretion as a function of time of culture, this increase was found under the control condition as well as on cells growing on the scaffold with no statistical differences between them. One of the reasons for the interest on chitosan-based biomaterials is the low cytotoxicity of chitosan. ${ }^{[4]}$ On the other hand, we have also previously demonstrated the in vitro biocompatibility and low toxicity of polyfumarates and chitosan-based materials. ${ }^{[23,45-48]}$ However, cross-linking improves the properties of the biomaterials, but most cross-linkers either cause undesirable changes to the functionality of the biopolymers or result in cytotoxicity. Although we used borax, which is considered a low cytotoxic cross-linker, we investigated the cytotoxicity of the biomaterial obtained. ${ }^{[16]}$ In this work, we used the monocyte-macrophage RAW264.7 cells, to demonstrate that the combination of these two materials using borax to obtain the new cross-linked material did not generate any toxicity.

Table 2. Nitric oxide (NO) production was determined by Griess' reaction using RAW 264.7 macrophages (Control condition: standard tissue culture dishes. Results are expressed as the media \pm SEM, $n=6)$.

\begin{tabular}{lccc}
\hline & \multicolumn{3}{c}{ No $\left[\mathbf{n m o l ~ \mathbf { ~ L } ^ { - 1 } ]}\right.$} \\
\cline { 2 - 4 } & $\mathbf{2 4 \mathbf { h }}$ & $\mathbf{4 8 ~ h}$ & $\mathbf{7 2 ~ h}$ \\
\hline Control & $0,014 \pm 0,017$ & $0,262 \pm 0,074$ & $0,096 \pm 0,041$ \\
PFVH-CHI-B & $0,034 \pm 0,034$ & $0,389 \pm 0,148$ & $0,035 \pm 0,023$
\end{tabular}

Table 3. Cytokine production: IL1 $\beta$ and TNF $\alpha$ production was evaluated as markers of cytotoxicty (Control condition: standard tissue culture dishes. Results are expressed as the media $\pm \mathrm{SEM}, n=3$ ).

\begin{tabular}{lccccc}
\hline & \multicolumn{2}{c}{ IL1 $\beta\left[\mathrm{pg} \mathrm{mL}^{-1}\right]$} & & \multicolumn{2}{c}{ TNF $\alpha\left[\mathrm{pg} \mathrm{mL} \mathrm{mL}^{-1}\right]$} \\
\cline { 2 - 3 } \cline { 5 - 6 } Time [d] & Control & PFVH-CHI-B & & Control & PFVH-CHI-B \\
\hline 2 & $0.33 \pm 0.31$ & $0.28 \pm 0.05$ & & $32 \pm 6$ & $20 \pm 5$ \\
7 & $1.57 \pm 0.31$ & $1.92 \pm 0.37$ & & $30 \pm 7$ & $14 \pm 2$ \\
13 & $1.11 \pm 0.20$ & $1.78 \pm 0.26$ & $64 \pm 12$ & $56 \pm 1$
\end{tabular}

\section{Conclusions}

In conclusion we have obtained and characterized a borax cross-linked chitosan and vinyl acetate-fumarate copolymer-based biomaterial for osteochondral tissue engineering. This cross-linked biomaterial could be considered as a hydrogel based in its swelling behavior with mechanical properties between bone and cartilage tissue. Biocompatibility studies demonstrate the versatility of this material since it allows BMPC osteogenic development and supports primary chondrocytes growth and extracellular matrix deposition. Moreover, we demonstrated that this cross-linked scaffold presented low cytotoxicity and could be degraded slowly to meet the requirements of osteochondral tissue reparation.

Acknowledgements: M.L.L. is a Doctoral Fellow of Universidad Nacional de La Plata, M.S.M. is Investigador Adjunto CONICET, A.M.C. is Investigador Principal CIC-PBA, and M.S.C. is Professor DE of UNLP. The authors thank Ms. María Cecilia Moreno for the revision and improvement of the language of this paper. This work was in part supported by PICT2012-0053 of ANPCYT and Research Proyect -11/X644 - UNLP.

Received: May 27, 2016; Revised: September 30, 2016; Published online: November 28, 2016; DOI: 10.1002/mabi.201600219

Keywords: biocompatibility; bone; cartilage; chitosan; crosslinked polymer; polyfumarate; tissue engineering

[1] S. Panseri, A. Russo, C. Cunha, A. Bondi, A. Di Martino, S. Patella, E. Kon, Knee Surg. Sports Traumatol. Arthrosc. 2012, 20, 1182.

[2] G. Musumeci, P. Castrogiovanni, R. Leonardi, F. M. Trovato, M. A. Szychlinska, A. Di Giunta, C. Loreto, S. Castorina, World J. Orthop. 2014, 5, 80.

[3] E. B. Hunziker, K. Lippuner, M. J. Keel, N. Shintani, Osteoarthritis Cartilage 2015, 23, 334.

[4] S. Bose, M. Roy, A. Bandyopadhyay, Trends Biotechnol. 2012, 30, 546 .

[5] M. E. Gomes, H. S. Azevedo, A. R. Moreira, V. Ella, M. Kellomaki, R. L. Reis, J. Tissue Eng. Regener. Med. 2008, 2, 243.

[6] X. Li, Y. Li, Y. Zuo, D. Ou, Y. Liu, T. Chen, N. Jiang, H. Li, J. Li, J. Biomed. Mater. Res., Part A 2015, 103, 3226.

[7] D. W. Jenkins, S. M. Hudson, Chem. Rev. 2001, 101, 3245. 
[8] Y. J. Park, K. H. Kim, J. Y. Lee, Y. Ku, S. J. Lee, B. M. Min, C. P. Chung, Biotechnol. Appl. Biochem. 2006, 43, 17.

[9] M. H. Cho, K. S. Kim, H. H. Ahn, M. S. Kim, S. H. Kim, G. Khang, B. Lee, H. B. Lee, Tissue Eng., Part A 2008, 14, 1099.

[10] I. Y. Kim, S. J. Seo, H. S. Moon, M. K. Yoo, I. Y. Park, B. C. Kim, C. S. Cho, Biotechnol. Adv. 2008, 26, 1.

[11] P. Ghosh, A. P. Rameshbabu, S. Dhara, Langmuir 2014, 30, 8442.

[12] C. H. Chen, F. Y. Wang, C. F. Mao, W. T. Liao, C. D. Hsieh, Int. J. Biol. Macromol. 2008, 43, 37.

[13] S. Liang, L. Liu, O. Huang, K. L. Yam, Carbohydr. Polym. 2009, 77, 718.

[14] V. M. Bispo, A. A. Mansur, E. F. Barbosa-Stancioli, H. S. Mansur, J. Biomed. Nanotechnol. 2010, 6, 166.

[15] C. Li, L. Wang, Z. Yang, G. Kim, H. Chen, Z. Ge, J. Biomater. Sci., Polym. Ed. 2012, 23, 405.

[16] J. Maitra, V. K. Shukla, Am. J. Polym. Sci. 2014, 4, 25.

[17] A. Radhakrishnan, G. M. Jose, M. Kurup, Prog. Biomater. 2015, 4, 101.

[18] M. S. Cortizo, J. Appl. Polym. Sci. 2007, 103, 3785.

[19] J. Brugnerotto, J. Lizardi, F. M. Goycoolea, W. Argüelles Monal, J. Desbriéres, M. Rinaudo, Polymer 2001, 42, 3569.

[20] M. Rinaudo, M. Milas, P. LeDung, Int. J. Biol. Macromol. 1993, 15, 281.

[21] T. G. Oberti, J. L. Alessandrini, M. S. Cortizo, J. Therm. Anal. Calorim. 2012, 109, 1525.

[22] B. Sreedhar, M. Sairam, D. Chattopadhyay, P. Rathnam, D. Rao, J. App. Polym. Sci. 2005, 96, 1313.

[23] J. M. Fernandez, M. S. Molinuevo, M. S. Cortizo, A. M. Cortizo, J. Tissue Eng. Regener. Med. 2011, 5, e126.

[24] M. Pasqualone, T. G. Oberti, H. A. Andreetta, M. S. Cortizo, J. Mater. Sci.: Mater. Med. 2013, 24, 1683.

[25] P. L. Ritger, N. A. Peppas, J. Controlled Release 1987, 5, 23.

[26] F. Ganji, S. Vasheghani-Farahani, E. Vasheghani-Farahani, Iran. Polym. J. 2010, 19, 375.

[27] M. S. Molinuevo, L. Schurman, A. D. McCarthy, A. M. Cortizo, M. J. Tolosa, M. V. Gangoiti, V. Arnol, C. Sedlinsky, J. Bone Miner. Res. 2010, 25, 211.

[28] W. Oiu, M. M. Murray, S. Shortkroff, C. R. Lee, S. D. Martin, M. Spector, Wound Repair Regener. 2000, 8, 383.

[29] T. Otsu, A. Matsumoto, K. Shiraishi, N. Amaya, Y. Koinuma, J. Polym. Sci., Part A: Polym. Chem. 1992, 30, 1559.
[30] A. H. Al-Arbash, F. A. Elsagheer, A. A. M. Ali, M. Z. Elsabee, J. Polym. Sci., Part A: Polym. Chem. 1999, 37, 1839.

[31] T. Otsu, H. Minai, N. Toyoda, T. Yasuhara, Makromol. Chem. 1985, 12, 133.

[32] J. G. Dawber, S. I. E. Green, J. Chem. Soc., Faraday Trans. 1986, $82,3407$.

[33] I. Uslu, H. Celikkan, O. Atakol, M. L. Aksu, Hacettepe J. Biol. Chem. 2008, 36, 117.

[34] S. Hirano, H. Tsuchida, N. Nagao, Biomaterials 1989, 10, 574.

[35] A. R. Sarasam, R. K. Krishnaswamy, S. V. Madihally, Biomacromolecules 2006, 7, 1131.

[36] T. A. Kelly, B. L. Roach, Z. D. Weidner, C. R. Mackenzie-Smith, G. D. O'Connell, E. G. Lima, A. M. Stoker, J. L. Cook, G. A. Ateshian, C. T. Hung, J. Biomech. 2013, 46, 1784.

[37] R. Scaffaro, F. Lopresti, L. Botta, S. Rigogliuso, G. Ghersi, J. Mech. Behav. Biomed. Mater. 2016, 54, 8.

[38] E. S. Costa-Júnior, E. F. Barbosa-Stancioli, A. A. P. Mansur, W. L. Vasconcelos, H. S. Mansur, Carbohydr. Polym. 2009, 76, 472.

[39] A. Hashemi Doulabi, H. Mirzadeh, M. Imani, N. Samadi, Carbohydr. Polym. 2013, 92, 48.

[40] S. Elder, A. Gottipati, H. Zelenka, J. Bumgardner, Open Orthop. J. 2013, 7, 275.

[41] A. Ferrand, S. Eap, L. Richert, S. Lemoine, D. Kalaskar, S. Demoustier-Champagne, H. Atmani, Y. Mely, F. Fioretti, G. Schlatter, L. Kuhn, G. Ladam, N. Benkirane-Jessel, Macromol. Biosci. 2014, 14, 45.

[42] A. Gupta, S. Bhat, P. R. Jagdale, B. P. Chaudhari, L. Lidgren, K. C. Gupta, A. Kumar, Tissue Eng., Part A 2014, 20, 3101.

[43] W. E. Muller, M. Neufurth, S. Wang, E. Tolba, H. C. Schroder, X. Wang, Eur. Cells Mater. 2016, 31, 174.

[44] F. Croisier, C. Jerome, Eur. Polym. J. 2013, 49, 780.

[45] M. S. Cortizo, M. S. Molinuevo, A. M. Cortizo, J. Tissue Eng. Regener. Med. 2008, 2, 33.

[46] J. M. Fernandez, M. S. Molinuevo, A. M. Cortizo, A. D. McCarthy, M. S. Cortizo, J. Biomater. Sci., Polym. Ed. 2010, 21, 1297.

[47] J. M. Fernández, M. S. Cortizo, A. M. Cortizo, J. Biomater. Tissue Eng. 2014, 4, 227.

[48] M. S. Belluzo, L. F. Medina, A. M. Cortizo, M. S. Cortizo, Ultrason. Sonochem. 2016, 30, 1. 